\title{
Naturheilverfahren auf dem Weg in die Zukunft
}

\begin{abstract}
— In den letzten Jahren hat die Akzeptanz von Komplementärmedizin in den Industriestaaten deutlich zugenommen. Das National Center for Complementary and Alternative Medicine (NCCAM), ein Teil der National Institutes of Health (NIH), hat hier mit seinen Forschungsprogrammen einen wesentlichen Beitrag geleistet.
\end{abstract}

\section{Anregung der Selbstheilungskräfte}

Die Definition der Naturheilkunde durch die Bundesärztekammer von 2004 trifft den Kern: Der Anwendung von Naturheilverfahren liegt ein ganzheitliches Medizinverständnis zugrunde, das ressourcenorientiert und auf Nachhaltigkeit bedacht ist. Im Rahmen der individualisierten und adaptionsorientierten, typischerweise multimodalen Behandlungskonzeption werden physiologische und psychologische Regulationsmechanismen genutzt. Therapieziele sind die Anregung der Selbstheilungskräfte durch naturgegebene Einwirkungen einschließlich gezielter unspezifischer und spezifischer systemischer und lokaler Reize sowie die Anleitung zur Übernahme von Eigenverantwortung durch den Patienten.

Die Bundesärztekammer hat zudem im Jahr 2007 naturheilkundliche Therapiemaßnahmen explizit zum Bestandteil der Weiterbildungsordnung für die Gebietsbezeichnung "Physikalische und Rehabilitative Medizin" gemacht. Auch für andere Verfahren aus der Komplementärmedizin, z. B. für die Akupunktur, wurden in den letzten Jahren Curricula erarbeitet.

\section{Probleme klinischer Studien}

Global sind große Anstrengungen unternommen worden, Methoden der Komple- mentärmedizin bei den postulierten Indikationen im Sinne der Evidenzbasierung abzusichern sowie physiologische und pharmakologische Wirkmechanismen aufzuklären. Die Ergebnisse klinischer Studien wurden jedoch oft sehr kritisch analysiert, ohne zu berücksichtigen, dass die Methodik der Evidenzbasierung ursprünglich für chemisch definierte Pharmaka entwickelt wurde und damit nur partiell für andere medizinische Verfahren geeignet ist. Die meisten komplementärmedizinischen Verfahren sind jedoch nicht pharmakologisch. Sie sind zudem dadurch gekennzeichnet, dass für sie in der Regel kein Patentschutz möglich ist. Sie sind oft sehr preiswert, die Kosten werden aber in Deutschland nicht von den gesetzlichen Krankenkassen getragen.

\section{Unsachliche Diskussionen}

Außerdem muss die Qualität überwiegend über die Anwender gesichert werden. Die Verfahren wirken eher selten sofort, die behandelbaren Indikationen stehen gegenwärtig oft nicht im Fokus der konventionellen Medizin. Da Forschung aber viel Geld kostet, das im Bereich der Komplementärmedizin aus den genannten Gründen nicht vorhanden ist, ist es leicht nachzuvollziehen, dass in vielen Bereichen noch qualitativ gute Studien fehlen. In einigen Ländern wie China, Kanada oder den USA stehen hierfür seit einigen Jahren erhebliche öffentliche Mittel zur Verfügung, in Deutschland dagegen sind entsprechend ausgewiesene Forschungsmittel bisher kaum vorhanden.

Bei der öffentlichen Diskussion um die Komplementärmedizin fällt auf, dass sie oft von Personen geführt wird, deren Ex-

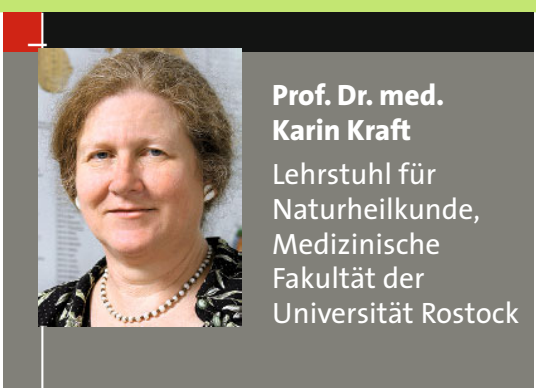

pertise fragwürdig ist, die Experten aber nicht gehört werden. Dies hat in der Vergangenheit zu Fehlinterpretationen geführt, die den sachlichen Umgang mit dieser Form der Medizin behindert haben.

\section{Ergänzung zur} konventionellen Medizin

Tatsächlich können diese Verfahren, die bei den Patienten wegen ihrer guten Verträglichkeit und teilweise auch nachhaltigen Wirkung äußerst beliebt sind, bei vielen chronischen Erkrankungen ergänzend zur konventionellen Medizin eingesetzt werden. Diese Form der Anwendung wird u. a. in China, Indien, Kanada und den USA offiziell unterstützt und als integrative Medizin bezeichnet. Auch in Deutschland werden zunehmend komplementärmedizinische, v. a. naturheilkundliche Verfahren in den Leitlinien wissenschaftlicher Gesellschaften erwähnt und auch z. T. empfohlen. Es bleibt zu wünschen, dass sie im deutschen Gesundheitswesen eine größere Rolle spielen als bisher.

\section{Neue MMW-Serie}

Der folgende Beitrag zur Phytotherapie bei Husten bildet den Auftakt der neuen Serie ,Sprechstunde Naturheilkunde“. Hier erfahren Sie, bei welchen Indikationen Sie Ihren Patienten welche naturheilkundlichen Therapieverfahren ergänzend anbieten können. Federführende Autoren sind Prof. Dr. Karin Kraft, Universität Rostock, und PD Dr. André-Michael Beer, Universitätsklinik Bochum. 